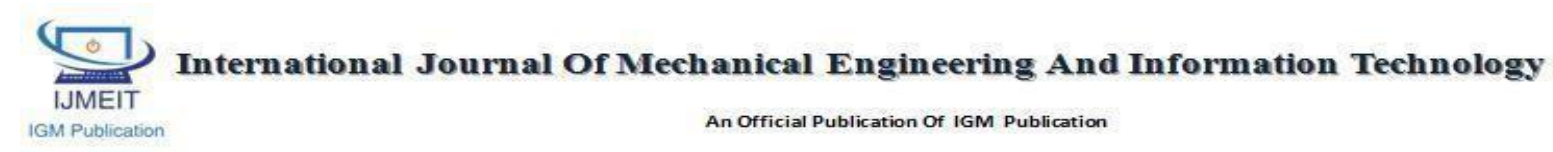

\title{
Enhancement of Adhoc Wireless network Security by Customized Encryption Technology \& using Multilayer of Security
}

\author{
Authors \\ Shokeen $^{1}$, Swati Gupta ${ }^{2}$ \\ ${ }^{1}$ M.Tech Rayat-Bahra Royal Institute of Management \& Technology \\ ${ }^{2}$ Assistant Professor Rayat-Bahra Royal Institute of Management \& Technology \\ Email-shokeentaneja06@gmail.com
}

\begin{abstract}
:
Adhoc Wireless computing proposes new ways to provide services. These pioneering technical \& pricing opportunities bring changes within way business operated. Lack of security is only barrier within wide adoption of cloud computing. The rapid growth of Wireless computing has brought many security challenges for users. Wireless computing offers many benefits, but it also is vulnerable to threats.
\end{abstract}

\section{INTRODUCTION}

A wireless network is any type of computer network which is using wireless data connections to connect network nodes. Wireless networking is a method by which domestic, telecommunications networks \& enterprise installations avoid costly process of introducing cables in a building. Because a connection among different equipment locations. Wireless telecommunications networks are generally implemented \& administered using radio communication. Such implementation takes place at physical level of Open System Interface model network structure. The examples of wireless networks consist of cell phone networks, Wireless local networks, wireless sensor networks, satellite communication networks, \& terrestrial microwave networks.

Terrestrial microwave - Terrestrial microwave communication usually make utilization of Earthbased transmitters \& receivers resembling satellite dishes. Terrestrial microwaves are within low gigahertz range, which limits all communications to line-of-sight. Relay stations are spaced approximately forty eight km (thirty mi) apart.

Communications satellites - Satellites communicate via microwave radio waves, which are not deflected by Earth's atmosphere. The satellites are stationed within space, typically within geosynchronous orbit $35,400 \mathrm{~km}$ (22,000 mi) above equator. These Earth-orbiting systems are capable of receiving $\&$ relaying voice, data, \& TV signals.

Cellular \& PCS systems make use of make several radio communications technologies. The systems divide region covered into multiple geographic areas. Every area has a low-power transmitter or radio relay antenna device to relay calls from one area to next area.

Radio \& spread spectrum technologies Wireless local area networks use a high-frequency radio technology is same as digital cellular \& a low-frequency radio technology. Wireless LANs use spread spectrum technology to enable communication among many devices within a limited area. IEEE 802.11 defines a common flavor of open-standards wireless radio-wave technologies are known as Wifi.

Free-space optical communication uses for visible or invisible light for communications. In most cases, line-of-sight propagation is used, which limits physical positioning of communicating devices. 


\section{TYPES OF WIRELESS NETWORKS}

\section{Wire-less PAN}

Wireless is a personal area networks interconnect devices within a relatively small area, that is comely with a person's reach. example, both Bluetooth radio \& invisible infrared light provides a WPAN for interconnecting a headset to a laptop. ZigBee also supports WPAN applications. Wi-Fi PANs are becoming common place as equipment designers are starting to integrate $\mathrm{Wi}-\mathrm{Fi}$ into the variety of costumer electronic devices. Intel My Wi-Fi \& Windows seven virtual Wi-Fi capabilities have made Wi-Fi PANs simpler \& easier to setup \& configure.

Wireless LAN

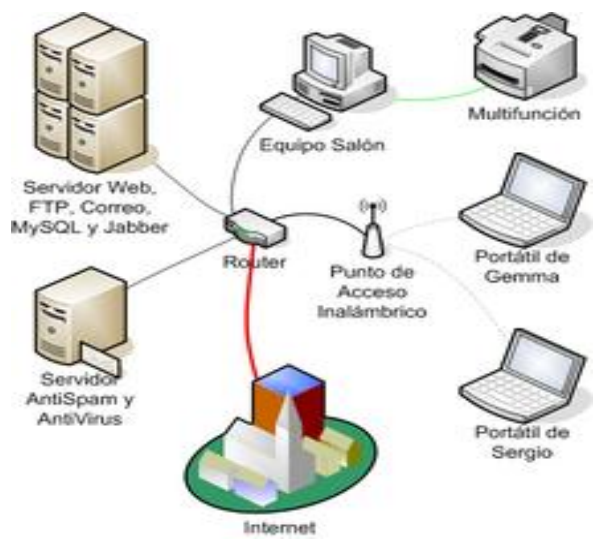

Fig 1. Wireless Local area Network

Wireless Land area networks is often used for connecting to local resources \& to Internet A wireless local area network (WLAN) links are two and more than two devices over short distance using a wireless distribution method, usually providing a connection by an access point for internet accessing. The use for spread-spectrum or OFDM technologies could allow the users to move within a local coverage area, \& still remain connected to network.

Products using IEEE 802.11 WLAN standards have been marketed under the brand name of WiFi. Fixed wireless technology implementation point-to-point links among computers/networks at two locations at different places, often using for dedicated microwave/ modulated laser light beams over line of sight paths. It is often used within cities to connect networks within two/more buildings without installing a wired link.

\section{Wireless mesh network}

Wireless mesh network is wireless network made up for radio nodes organized within a mesh topology. Each node forwards messages on behalf of other nodes. Mesh networks could self-heal, automatically re-routing a node which has lost its power.

\section{Wireless MAN}

Wireless metropolitan area networks are a type of wireless network which would connect several wireless LANs. WiMAX is a type of Wireless MAN \& is described by IEEE 802.16 standard.

\section{Wireless WAN}

Wireless WAN is wireless networks is that typical covered large areas, like between neighbouring towns \& cities, or city \& suburb. These networks could be used to connect branch offices of business or as a public internet access system. Wireless connections among access points are usually point to point microwave links using parabolic dishes on $2.4 \mathrm{GHz}$ band, rather than omnidirectional antennas used with smaller networks. A typical system consists of base station gateways, access points \& wireless bridging relays. Other configurations are mesh systems where every access point acts as a relay also. When combined with renewable energy systems like photovoltaic solar panels or wind systems they could be stand alone systems.

Global area network

A global area network is used for supporting mobile across a arbitrary are number of the wireless LANs, satellite coveraged areas, etc. The key challenged with in mobile network is handing off user communications from one local coverage area to next. In IEEE Project 802, this consists of a succession of terrestrial wireless LANs.

\section{Space network}

Space networks are networks used for communication between spacecraft, usually within vicinity of Earth. Example of this is NASA's Space Network. 


\section{CHALLENGES}

Lack of security is only barrier within wide adoption of cloud computing. The rapid growth for cloud computing have buy more security challenges for users. Cloud computing offers many benefits, but it also is vulnerable to threats. One of main threat exist today is problem of unauthorized users or entities. For avoiding this problem new technique is developed within this cloud computing is that data owners could share their outsourced data with a large number of users, who might want to retrieve certain specific data files they are interested within during a given session.

\section{EXISTING IMPLEMENTATION \\ Cryptography \\ Encryption Steps:-}

- Data would be encrypted with in Rivers Shamir Adleman algorithm for example RSA.

- A digital envelope is sent to receiver having cipher text encrypted.

\section{Decryption Steps:-}

The Decryption of message received from sender's side would occour as follow:

- Digital envelope would reach receiver's side.

- Digital envelope would be opened to get encrypted data \& decrypt using its own private key with RSA algorithm \& receiver get secret picture.

- Thus receiver would get plain text.

\section{Socket implementation after Cryptography}

Here we would create our server \& client communication protocol using own port using socket programming.

1. First step is to create server side port using following algorithm

- Create ServerSocket object using our own port 6666.

- Accept client request using Server socket object.

- Receive data from client within form of input data stream object.
- Convert data stream object to string

- Input data stream is within form of cipher data decrypted are using proposed algorithm.

- The Close Connection

2. Second step is to create Client side interface to connect to server.

- Create ServerSocket object using our own port 6666 to connect to server

- Encrypt data before sending.

- Send data using data output stream object.

- Clean output buffer.

- Close connection.

\section{PROBLEM STATEMENT}

There was problem with existing security system. There was security threat by crypto analyst. Crypto analyst is person who could access convert encrypted data to decrypted form using this cracking techniques.

\section{OBJECTIVE OF RESEARCH}

The main objective of research is to secure the data by integrating IP filter and OTP mechanism to enhance the security of cryptography based wireless network.

\section{PROPOSED MODEL}

In proposed model there would be triple layered security

1. Security layer 1 would be customized cryptography algorithm of AES to enhance security.

2. Security layer 2 would drop packets from unauthentic IP addresses.

3. Security layer 3 would authenticate user by providing login password security at application layer.

4. Security would be enhanced using one time password also that becomes useless after using one time.

5. In this way we will secure wireless network from external attacks and authentic access. 


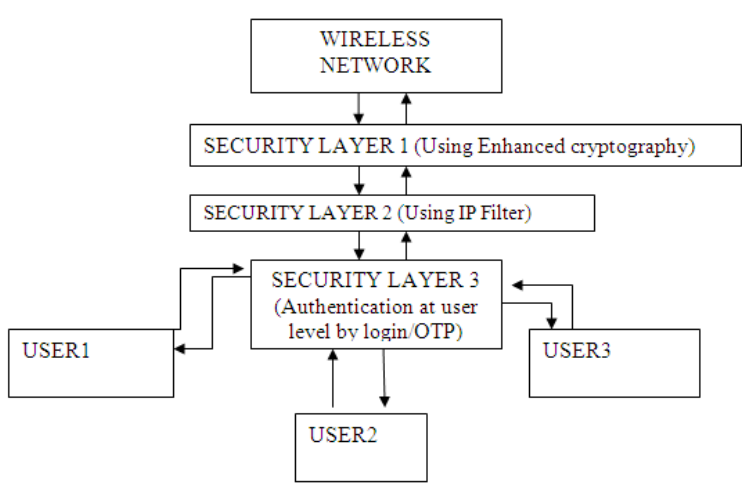

Fig. 2 Triple Layer Security

\section{OTP Generation}

One time password would be generated randomly by Math.random() function in java. Each and every time the complex OTP number could be generated to be used during decryption.

Using Math. random() double random = Math. random ()$* 50+1$; or int random $=($ int $)($ Math. random ()$* 50+1) ; \ldots$

Using Random class in Java. Random rand = new Random(); int value = rand .nextInt(50); This will give value from 0 to 49 . For 1 to 50: rand.nextInt $(50)+1$

\section{IP Filter}

Centralized database of IP address would be created on centralized server and decryption request from authentic IP would be accepted. If IP is not found in database or its status is 0 then Decryption would not be allowed.

Data in case of Fiber optics

\section{Table 1 Data in case of Fiber optics}

\begin{tabular}{|l|l|l|l|l|}
\hline Sno & Security_Level & H & L & Avg \\
\hline 1 & Layer1(cr) & 20 & 40 & 30 \\
\hline 2 & Layer2(ip) & 15 & 30 & 22.5 \\
\hline 3 & Layer3(otp) & 10 & 20 & 15 \\
\hline 4 & L1+L2 & 40 & 80 & 60 \\
\hline 5 & L1+L3 & 35 & 70 & 52.5 \\
\hline 6 & L2+L3 & 30 & 60 & 45 \\
\hline 7 & L1+L2+L3 (slow_net) & 55 & 110 & 82.5 \\
\hline 8 & L1+L2+L3(avg_net) & 50 & 100 & 75 \\
\hline 9 & L1+L2+L3(High_net) & 48 & 96 & 72 \\
\hline 10 & L1+L2(avg_net) & 45 & 90 & 67.5 \\
\hline 11 & L1+L3(avg_net) & 40 & 80 & 60 \\
\hline 12 & L2+L3(avg_net) & 35 & 70 & 52.5 \\
\hline
\end{tabular}

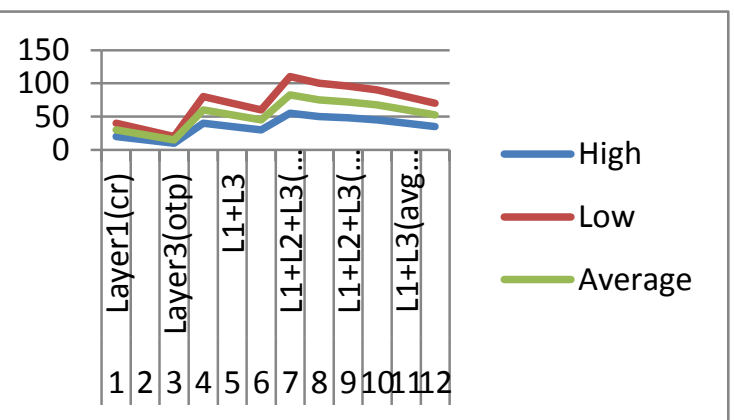

Fig 3. Analysis of transmission speed of packet in case of Fiber optics

Table 2 Data in case of Coaxial Cable

\begin{tabular}{|l|l|l|l|l|}
\hline Sn. & Security_Level & H & L & Avg \\
\hline 1 & Layer1(cr) & 25 & 50 & 37.5 \\
\hline 2 & Layer2(ip) & 20 & 40 & 30 \\
\hline 3 & Layer3(otp) & 15 & 30 & 22.5 \\
\hline 4 & L1+L2 & 45 & 90 & 67.5 \\
\hline 5 & L1+L3 & 40 & 80 & 60 \\
\hline 6 & L2+L3 & 35 & 70 & 52.5 \\
\hline 7 & L1+L2+L3(slow_net) & 60 & 120 & 90 \\
\hline 8 & L1+L2+L3(avg_net) & 55 & 110 & 82.5 \\
\hline 9 & L1+L2+L3(High_net) & 53 & 106 & 79.5 \\
\hline 10 & L1+L2(avg_net) & 50 & 100 & 75 \\
\hline 11 & L1+L3(avg_net) & 45 & 90 & 67.5 \\
\hline 12 & L2+L3(avg_net) & 40 & 80 & 60 \\
\hline
\end{tabular}

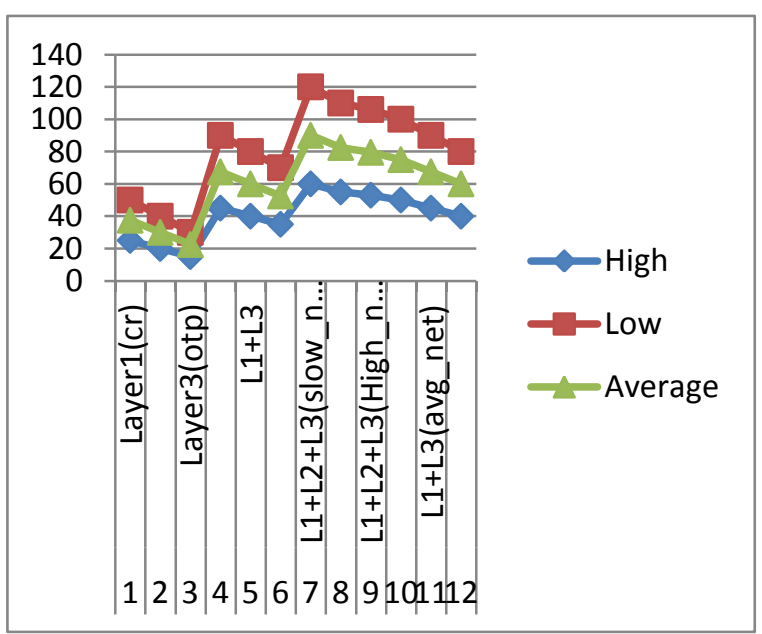

Fig 4. Analysis of transmission speed of packet in case of Coaxial Cable 
Table 3 Data in case of Twisted Cable

\begin{tabular}{|l|l|l|l|l|}
\hline Sno & Security_Level & H & L & Avg \\
\hline 1 & Layer1(cr) & 30 & 60 & 45 \\
\hline 2 & Layer2(ip) & 25 & 50 & 37.5 \\
\hline 3 & Layer3(otp) & 20 & 40 & 30 \\
\hline 4 & L1+L2 & 50 & 100 & 75 \\
\hline 5 & L1+L3 & 45 & 90 & 67.5 \\
\hline 6 & L2+L3 & 40 & 80 & 60 \\
\hline 7 & L1+L2+L3(slow_net) & 65 & 130 & 97.5 \\
\hline 8 & L1+L2+L3(avg_net) & 60 & 120 & 90 \\
\hline 9 & L1+L2+L3(High_net) & 58 & 116 & 87 \\
\hline 10 & L1+L2(avg_net) & 55 & 110 & 82.5 \\
\hline 11 & L1+L3(avg_net) & 50 & 100 & 75 \\
\hline 12 & L2+L3(avg_net) & 45 & 90 & 67.5 \\
\hline
\end{tabular}

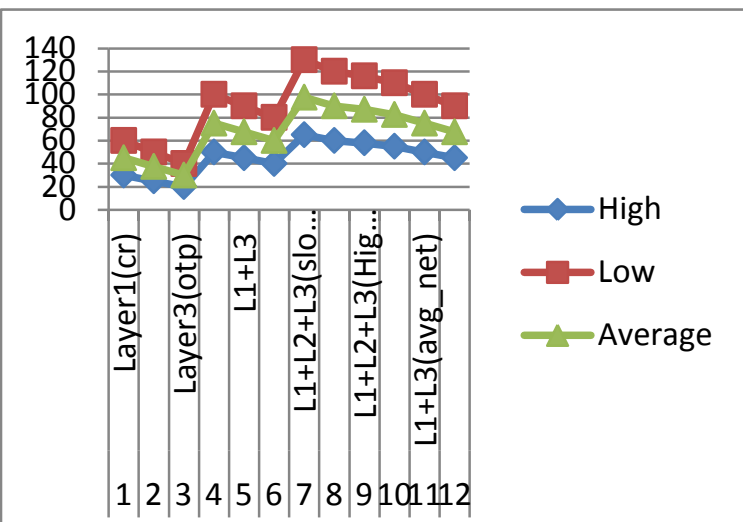

Fig 5 Analysis of transmission speed of packet in case of Twisted Cable

\section{Table 4 Data in case of Wireless Network}

\begin{tabular}{|l|l|l|l|l|}
\hline Sr. No. & Security_Level & H & L & Avg \\
\hline 1 & Layer1(cr) & 35 & 70 & 52.5 \\
\hline 2 & Layer2(ip) & 30 & 60 & 45 \\
\hline 3 & Layer3(otp) & 25 & 50 & 37.5 \\
\hline 4 & L1+L2 & 55 & 110 & 82.5 \\
\hline 5 & L1+L3 & 50 & 100 & 75 \\
\hline 6 & L2+L3 & 45 & 90 & 67.5 \\
\hline 7 & L1+L2+L3(slow_net) & 70 & 140 & 105 \\
\hline 8 & L1+L2+L3(avg_net) & 65 & 130 & 97.5 \\
\hline 9 & L1+L2+L3(High_net) & 63 & 126 & 94.5 \\
\hline 10 & L1+L2(avg_net) & 60 & 120 & 90 \\
\hline 11 & L1+L3(avg_net) & 60 & 120 & 90 \\
\hline 12 & L2+L3(avg_net) & 50 & 100 & 75 \\
\hline
\end{tabular}

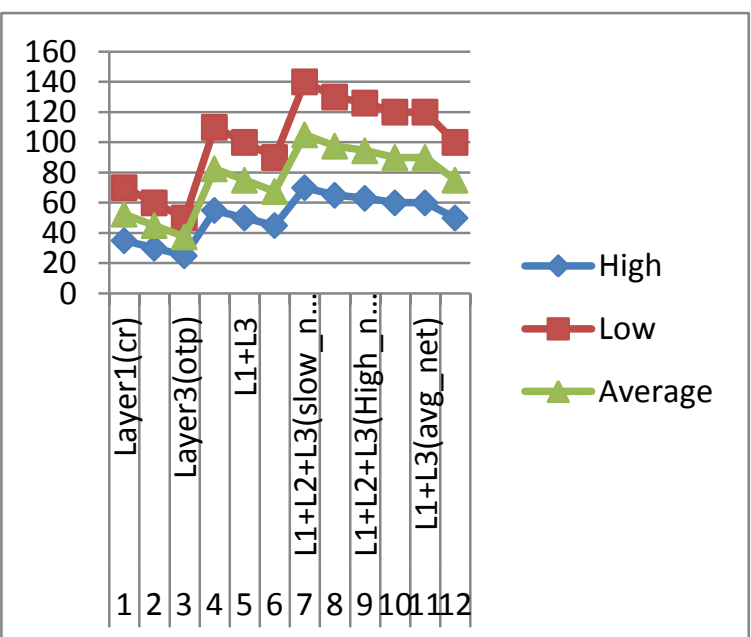

Fig 6 Analysis of transmission speed of packet in case of Adhoc Wireless network

\section{CONCLUSION \& FUTURE SCOPE}

We have enhanced security by enhancing encryption algorithm. Here we have also defined our own ports for server $\&$ client $\&$ defined new rules for encryption \& decryption \& involved multiple layer of security like IP Filter \& OTP code this would definitely improve security mechanism within Wireless computing environment.

Future Analysis could be made on different network and topology. Additional security layers may be added to enhance security.

\section{REFERENCES}

1. David Pointcheval, Olivier Blazy, New Smooth Projective Hash Functions \& OneRound Authenticated Key Exchange(18_22 august 2013, Santa Barbara, California, USA), SpringerVerlag, LNCS 8042, pages 449_475.

2. David Pointcheval, Olivier Blazy, Effcient UC-Secure Authenticated Key-Exchange for Algebraic Languages(26 February - 1 March 2013, Nara, Japan)), 16th International Conference on Practice \& Theory within Public-Key Cryptography (PKC '13)Springer-Verlag, Kaoru Kurosawa Ed., Springer-Verlag, 2013.

3. David Pointcheval, Password-based Authenticated Key Exchange. (21-23 may 
2012, Darmstadt, Germany)Springer-

Verlag, LNCS 7293, pages 390-397.

4. David Pointcheval, Michel Abdalla, Contributory Password-Authenticated

Group Key Exchange with Join Capability, (February 14-18, 2011, San Francisco, CA, USA), A. Kiayias Ed. Springer-Verlag, LNCS 6558, pages 142-160.

5. David Pointcheval, Xavier Boyen, Strong Cryptography from Weak Secrets, (3 - 6 may 2010, Stellenbosch, South Africa), D. Bernstein \& T. Lange Eds., SpringerVerlag, LNCS 6055, pages 297-315.

6. David Pointcheval, Michel Abdalla, Flexible Group Key Exchange with OnDemand Computation of Subgroup Keys, (3-6 May 2010, Stellenbosch, South Africa)), D. Bernstein \& T. Lange Eds., Springer-Verlag, LNCS 6055, pages 351368.

7. David Pointcheval, Michel Abdalla, Distributed Public-Key Cryptography from Weak Secrets, (18_20 march 2009, Irvine, CA, USA), S. Jarecki \& G. Tsudik Eds. Springer-Verlag, LNCS 5443, pages 139_159.

8. David Pointcheval, Michel Abdalla, Password- Authenticated Group Key Agreement with Adaptive Security \& Contributiveness, (21 - 25 june 2009, Gammarth, Tunisia) B. Preneel Ed., Springer-Verlag, LNCS 5580, pages 254271.

9. Rafael Álvarez, Leandro Tortosa, Analysis \& design of a secure key exchange scheme, Information Sciences 179 (2009), Elsevier 